\title{
Correlation of Ceruloplasmin with Biomarkers of Cardiac Remodelling and Myofibrosis in Patients with Acute Decompensated Heart Failure Referred to a Tertiary Nurse Lead Heart Failure Clinic
}

\author{
Filip Málek ${ }^{1,2 *}$, Dagmar Vondráková1,2, Oxana Komendová1, Dana Ř́ihová1, Jana Vránová2 \\ Lenka Sedláčková ${ }^{3}$, Táňa Andreasová ${ }^{1}$, Petr Neužil ${ }^{1}$ \\ ${ }^{1}$ Department of Cardiology, Na Homolce Hospital, Prague, Czech Republic \\ ${ }^{2}$ Third School of Medicine, Charles University, Prague, Czech Republic \\ ${ }^{3}$ Department of Clinical Biochemistry, Haematology and Immunology, Na Homolce Hospital, \\ Prague, Czech Republic \\ Email: "filip.malek@centrum.cz
}

Received 20 September 2015; accepted 8 November 2015; published 11 November 2015

Copyright (C) 2015 by authors and Scientific Research Publishing Inc.

This work is licensed under the Creative Commons Attribution International License (CC BY). http://creativecommons.org/licenses/by/4.0/

(c) (i) Open Access

\section{Abstract}

Background: Ceruloplasmin is an acute phase protein with plasma copper binding properties, and is a potent extracellular antioxidative enzyme. Inflammation and oxidative stress might explain the role of ceruloplasmin in the pathophysiology of heart failure. Study objective: The objective is to assess the correlation of ceruloplasmin levels with biomarkers of cardiac remodelling and myofibrosis in patients with acute decompensated heart failure. Patients and methods: Blood samples were taken and serum levels of soluble ST2, galectin-3, NT-proBNP and ceruloplasmin were analysed in 31 consecutive patients with systolic HF referred to tertiary care nurse lead heart failure clinic with acute decompensated CHF requiring i.v. diuretics. The mean patients' age was 68 years, mean left ventricular ejection fraction (LV EF) was $29 \%, 66 \%$ patients had ischemic aetilogy of CHF and $33 \%$ had atrial fibrillation. Results: The mean ceruloplasmin level was $0.243 \mathrm{~g} / \mathrm{l}$, mean galectin-3 level was $1.26 \mathrm{ng} / \mathrm{ml}$, mean sST2 level was $38.15 \mathrm{ng} / \mathrm{ml}$, and mean NT-proBNP was 1927 $\mathrm{pg} / \mathrm{ml}$. The ceruloplasmin level correlated with NT-proBNP $(r=0.58, p<0.05)$ and with $\mathrm{sST} 2(\mathrm{r}=$ $0.77, p<0.001)$, sST2 levels correlated significantly with NT-proBNP $(r=0.66, p<0.01)$. The ceruloplasmin level did not correlate with galectin-3 concentration. Conclusion: The ceruloplasmin level correlates with the biomarkers of cardiac remodelling (NT-proBNP, sST2), but not with the biomarker of myofibrosis (galectin-3). This finding supports the hypothesis of inflammatory re- 
sponse in acute decompensated heart failure.

\section{Keywords}

\section{Acute Decompensated Heart Failure, Ceruloplasmin, NT-proBNP, sST2, Galectin-3}

\section{Introduction}

Ceruloplasmin is an acute phase protein with plasma copper binding properties, and is considered to be one of the most potent extracellular antioxidative enzymes. Inflammation and oxidative stress might explain the role of ceruloplasmin in the pathophysiology of heart failure [1]. Historically, several epidemiologic studies showed the association of elevated ceruloplasmin levels with the risk of atherosclerosis, coronary heart disease, myocardial infarction and ischemic heart failure [2]-[5]. The Atherosclerosis Risk in Communities (ARIC) Study reveals the association of elevated ceruloplasmin levels with the risk of incident heart failure (HF), HF mortality and risk of cardiovascular disease (CVD) in healthy individuals [6]. In the patients with established chronic heart failure, ceruloplasmin levels correlated with the severity of heart and weakly with B-natriuretic peptide levels, but predicted all-cause mortality [7].

ST2 is a member of the interleukin-1 (IL-1) receptor family and consists of a trans-membrane ligand (STL2) and a soluble form (sST2). ST2 is released from cardiomyocytes and fibroblasts after mechanical strain. Soluble ST2 has been identified as a novel biomarker of cardiac stress and remodelling. In patients with acutely decompensated heart failure, the elevated sST2 concentrations predicted the risk of hospitalization and mortality, and this association was independent on natriuretic peptides levels and on other clinical variables [8] [9].

Galectin-3 is a protein secreted by activated macrophages and has a role in the development of fibrosis in injured tissues. Experimental studies showed increased galectin-3 secretion in the context of cardiac hypertrophy and heart failure models. Clinical studies identified galectin-3 to have additional prognostic information together with NT-proBNP in acute heart failure [10].

Relation of ceruloplasmin levels with the novel biomarkers of cardiac remodelling (sST2) and myofibrosis (galectin-3) has not been yet evaluated.

\section{Study Objective}

The aim of our study was to assess correlation of ceruloplasmin levels with biomarkers of cardiac remodelling (NT-proBNP, sST2) and biomarker of myofibrosis (Galectin-3) in patients with acute decompensated heart failure.

\section{Patients Population and Methods}

Blood samples were taken and serum levels of soluble ST2, galectin-3, NT-proBNP and ceruloplasmin were analysed in 31 consecutive patients, 22 men and eight women, with chronic systolic HF and left ventricular ejection fraction $\leq 35 \%$, referred to tertiary care nurse lead heart failure clinic with acute decompensated CHF requiring diuretic dose adjustment between $1^{\text {st }}$ January and $30^{\text {th }}$ June 2015 . Mean patients' age was 68 years (range 43 - 84 years), mean left ventricular ejection fraction (LV EF) was 29\%, ischemic aetilogy of CHF had 20 (66\%) patients and 10 (33\%) had chronic atrial fibrillation. Patients were treated according to Guidelines for the diagnosis and treatment of chronic heart failure. Diagnosis of CHF decompensation was based of physical examination showing signs of fluid overload and/or worsening dyspnea. Patients with acute coronary syndrome, pulmonary emboly, acute infection, know malignancy and chronic inflammatory or autoimmune disease were not included in the study.

Blood samples were taken on the day of clinic visit.

Ceruloplasmin determination was performed with the use of immunoturbidimetric assay.

Normal value range is $0.220-0.440 \mathrm{~g} / \mathrm{l}$ in healthy individuals.

The NT-proBNP analysis was performed with a commercially available chemiluminescence immunoassay for the determination of Human NT-proBNP: CLIA kit using Sandwich-CLIA as the method. The sST2 and galec- 
tin-3 was analyzed using a commercially available enzyme-linked immunosorbent assay kit. All biochemical analyses were realized on the analyser UnicelDxC 800 (Beckman Coulter Company, Germany) according to established methods.

An echocardiographic examination of the each patient was performed using a broadband transducer with a transmitting frequency from 1.7 to $4.0 \mathrm{MHz}$ on commercially available equipment (Vivid 7, GE, USA). Left ventricular ejection fraction was calculated by Simpson's method.

All patients' data were collected and transcribed to statistical testing. Spearman's correlation coefficient $r$ was used for statistical analysis. A $r$ value $>0.6$ and $p$ value $<0.05$ was considered significant.

The study was approved by Local Board Ethics Committee and conducted in accordance with the Declaration of Helsinki. All patients provided informed consent orally and in writing prior to enrollment.

\section{Results}

Results are shown in the table (Table 1). Mean ceruloplasmin level was $0.243 \mathrm{~g} / \mathrm{l}$, mean galectin-3 level was $1.26 \mathrm{ng} / \mathrm{ml}$, mean sST2 level was $38.15 \mathrm{ng} / \mathrm{ml}$, and mean NT-proBNP was $1927 \mathrm{pg} / \mathrm{ml}$. As shown in the table, mean ceruloplasmin value in our study was $0.243 \mathrm{~g} / \mathrm{l}$ and median $0.250 \mathrm{~g} / \mathrm{l}$ was in range of normal healthy subjects $(0.220-0.440 \mathrm{~g} / \mathrm{l})$.

Mean NT-proBNP level was $1927 \mathrm{pg} / \mathrm{ml}$, and median value was $1103 \mathrm{pg} / \mathrm{ml}$ (range 465 - $7856 \mathrm{pg} / \mathrm{ml}$ ), the values (mean and median) are above upper normal limit for acute heart failure (300 pg/ml).

Correlation of ceruloplasmin with biomarkers of cardiac remodelling and myofibrosis is shown in the table (Table 2). Ceruloplasmin level correlated with NT-proBNP ( $<0.05)$ and with sST2 ( $<0.001)$, sST2 levels corrrelated significantly with NT-proBNP $(\mathrm{p}<0.01)$. Ceruloplasmin level did not correlate with galectin-3 concentration.

\section{Discussion}

Ceruloplasmin is a glycoprotein synthetized in the liver and transports copper. Ceruloplasmin decreases nitric oxide (NO) bioavailibility through NO oxidase activity. Ceruloplasmin also enhances low-density lipoprotein (LDL) oxidation. Oxidized LDL has proatherogenic role and decreased NO availability contributes to endothelial dysfunction [11] [12]. Based on this information, several studies suggested association of elevated ceuroplasmin levels with CVD and HF [13] [14]. Ceruloplasmin is an acute phase protein too and its levels correlate with

Table 1. Results—echocardiography and laboratory parameters.

\begin{tabular}{ccccc}
\hline Parameter & Mean & Median & Minimum & Maximum \\
\hline LV EF \% & 28.89 & 30 & 16 & 35 \\
EDD LV mm & 63.39 & 62.50 & 53 & 80 \\
LA mm & 50.56 & 51 & 36 & 64 \\
BMI kg/m² & 29.62 & 29.20 & 21.10 & 45.70 \\
Potassium mmol/l & 4.27 & 4.20 & 3.60 & 5.10 \\
Sodium mmol/l & 137.28 & 137.0 & 131.0 & 143.0 \\
Urea mmol/l & 9.46 & 8.0 & 3.30 & 27.80 \\
Creatinine $\mu \mathrm{mol} / \mathrm{l}$ & 136.78 & 132.78 & 108.0 & 167.0 \\
eGFR ml/sec & 0.78 & 0.82 & 0.42 & 1.80 \\
Cp g/l & 0.243 & 0.250 & 0.140 & 0.320 \\
NT-proBNP pg/ml & 1927.25 & 1103.64 & 465.14 & 7856.53 \\
sST2 ng/ml & 38.15 & 31.50 & 14.60 & 112.00 \\
Gal-3 ng/ml & 1.266 & 1.021 & 0.212 & 3.220 \\
\hline
\end{tabular}

Table legend: LV EF-left ventricular ejection fraction, EDD-LV—left ventricular end diastolic diameter, LA—left atrial diameter, BMI — body mass index, eGFR — estimated glomerular filtration rate (MDRD formula), $\mathrm{Cp}$ —ceruloplasmin, NT-proBNP—N-terminal B-natriuretic peptide, Gal-3—galectin-3. 
Table 2. Correlation of ceruloplasmin with other heart failure biomarkers.

\begin{tabular}{cccc}
\hline Parameter & Parameter & Corellation coefficient & Level of significance \\
\hline sST2 & NT-proBNP & $r=0.66$ & $\mathrm{p}<0.01$ \\
Cp & sST2 & $r=0.77$ & $\mathrm{p}<0.001$ \\
Cp & NT-proBNP & $r=0.58$ & $\mathrm{p}<0.05$ \\
Cp & Gal-3 & $r=0.24$ & $\mathrm{p}=0.345$ \\
Gal -3 & NT-proBNP & $r=0.41$ & $\mathrm{p}=0.087$ \\
\hline
\end{tabular}

Table legend: $\mathrm{Cp} —$ ceruloplasmin, NT-proBNP—N-terminal B-natriuretic peptide, Gal-3—galectin-3.

other inflammatory markers associated with CVD, e.g.: high-sensitivity C-reactive protein (hs-CRP) [13]. In the contrary with previous observations, ceruloplasmin levels were weakly associated with CVD in the ARIC study [6]. In the ARIC study, ceruloplasmin levels were associated with incident HF after adjusting for hs-CRP, N-terminal B-natriuretic peptide (NT-proBNP) and high-sensitivity cardiac troponin T. The genetic part of the study brought significant result showing that ceruloplasmin gene on chromosome 3 was not associated with incident HF but the ceruloplasmin level was.

In the patients with established chronic HF, ceruloplasmin levels correlated with CRP levels and showed independent association with the severity of heart failure as assessed by New York Heart Association class in nonischemic cardiomyopathy [15].

Association of ceruloplasmin with other heart failure biomarkers was analyzed in several studies. In a study of patients with stable chronic HF, ceruloplasmin levels weakly correlated with B-type natriuretic peptide $(r=$ $0.187, \mathrm{p}<0.001)[7]$.

In the patients with acute decompensated heart failure, ceruloplasmin correlated with NT-proBNP levels in a small study evaluated association of heart failure severity with the level of metabolic liver function [16].

Correlation of ceruloplasmin levels with the novel heart failure biomarkers, e.g.: marker of remodelling sST2 and marker of myofibrosis galectin-3 in the patients with decompensated heart failure has not been systematicaly yet studied.

In our study, ceruloplasmin level correlates with biomarkes of cardiac remodelling:

In our study, ceruloplasmin level in the patients with acute decompensated heart failure with fluid overload correlates with biomarkers of cardiac remodelling: NT-proBNP and sST2. On the other hand, ceruloplasmin levels did not correlate with the biomarker of myofibrosis (galectin-3).

\section{Conclusion}

Our findings support the inflammatory response and oxidative stress hypothesis in acute decompensated heart failure. It is possible that ceruloplasmin decreases the availability of NO and enhances the reactive oxidant species formation and oxidative cell injury. The cell injury might be associated with the increased wall stress leading to increased myocyte production of biomarkers of remodelling: NT-proBNP and sST2, but not with the increased expression of galectin-3. Galectin-3 is a protein secreted by activated macrophages and has a role in development of fibrosis in injured tissues [17].

\section{Acknowledgements}

Approval for the study was granted by Local Board Ethics Committee Na Homolce Hospital. Study was supported by institutional grant Na Homolce Hospital, Prague, Czech Republic IG 140505.

\section{References}

[1] Mak, S. and Newton, G.E. (2001) The Oxidative Stress Hypothesis of Congestive Heart Failure. Chest, 120, 20352046.

[2] Reunanen, A., Knekt, P. and Aaran, P.K. (1992) Serum Ceruloplasmin Level and the Risk of Myocardial Infarction and Stroke. American Journal of Epidemiology, 136, 1082-1090.

[3] Mänttäri, M., Manninen, V., Huttunen, V.K., et al. (1994) Serum Ferritin and Ceruloplasmin as Coronary Risk Factors 
European Heart Journal, 15, 1599-1603.

[4] Klipstein-Grobusch, K., Grobbee, D.E., Koster, F.E., et al. (1999) Serum Ceruloplasmin as a Coronary Risk Factor in the Elderly. The Rotterdam Study British Journal of Nutrition, 81, 139-144.

[5] Ridker, P.M., Cushman, M., Stampfer, M.J., et al. (1997) Inflammation, Aspirin, and the Risk of Cardiovascular Disease in Apparently Healthy Men. New England Journal of Medicine, 336, 973-979. http://dx.doi.org/10.1056/NEJM199704033361401

[6] Dadu, R.T., Dodge, R., Nambi, V., et al. (2013) Ceruloplasmin and Heart Failure in the Atherosclerosis Risk in Communities Study. Circ Heart Fail, 6, 936-943. http://dx.doi.org/10.1161/CIRCHEARTFAILURE.113.000270

[7] Hammadah, M., Fan, Y., Wu, Y., et al. (2014) Prognostic Value of Elevated Serum Ceruloplasmin Levels in Patients with Heart Failure. Journal of Cardiac Failure, 20, 946-952.

[8] Mueller, T., Dieplinger, B., Gegenhuber, A., et al. (2008) Increased Plasma Concentrations of Soluble ST2 Are Predictive for 1-Year Mortality in Patients with Acute Destabilized Heart Failure. Clinical Chemistry, 54, 752-756. http://dx.doi.org/10.1373/clinchem.2007.096560

[9] Manzano-Fernández, S., Mueller, T., Pascual-Figal, D., et al. (2011) Usefulness of Soluble Concentrations of Interleukin family member ST2 as Predictor of Mortality in Patients with Acutely Decompensated Heart Failure Relative to Left Ventricular Ejection Fraction. The American Journal of Cardiology, 107, 259-267. http://dx.doi.org/10.1016/j.amjcard.2010.09.011

[10] de Boer, R.A., Voors, A.A., Muntendam, P., et al. (2009) Galectin-3: A Novel Mediator of Heart Failure Development and Progression. European Journal of Heart Failure, 11, 811-817.

[11] Shiva, S., Wang, X., Ringwood, L.A., et al. (2006) Ceruloplasmin Is a No Oxidase and Nitrite Synthase That Determines Endocrine No Homeostasis. Nature Chemical Biology, 2, 486-493. http://dx.doi.org/10.1038/nchembio813

[12] Shukla, N., Maher, J., Masters, J., et al. (2006) Does Oxidative Stress Change Ceruloplasmin from a Protective to a Vasculopathic Factor? Atherosclerosis, 187, 238-250. http://dx.doi.org/10.1016/j.atherosclerosis.2005.11.035

[13] Tang, W.H., Wu, Y., Hartiala, J., Hazen, S.L., et al. (2012) Clinical and Genetic Association of Serum Ceruloplasmin with Cardiovascular Risk. Arteriosclerosis, Thrombosis, and Vascular Biology, 32, 516-522. http://dx.doi.org/10.1161/ATVBAHA.111.237040

[14] Engstrom, G., Hedblad, B., Tyden, P. and Lindgarde, F. (2009) Inflammation-Sensitive Plasma Proteins Are Associated with Increased Incidence of Heart Failure: A Population-Based Cohort Study. Atherosclerosis, 202, 617-622. http://dx.doi.org/10.1016/j.atherosclerosis.2008.05.038

[15] Xu, Y., Lin, H., Zhou, Y., et al. (2013) Ceruloplasmin and the Extent of Heart Failure in Ischemic and Nonischemic Cardiomyopathy Patients. Mediators of Inflammation, 2013, Article ID: 348145. http://dx.doi.org/10.1155/2013/348145

[16] Hendrichová, M., Málek, F., Kopřivová, H., et al. (2010) Correlation of NT-proBNP with Metabolic Liver Function as Assessed with ${ }^{13} \mathrm{C}$-Methacetin Breath Test in Patients with Acute Decompensated Heart Failure. International Journal of Cardiology, 144, 321-322. http://dx.doi.org/10.1016/j.ijcard.2009.03.022

[17] Sharma, U.C., Pokharel, S., van Brakel, T.J., et al. (2004) Galectin-3 Marks Activated Macrophages in FailurePronehy Pertrophied Hearts and Contributes to Cardiac Dysfunction. Circulation, 110, 3121-3128. http://dx.doi.org/10.1161/01.CIR.0000147181.65298.4D 